\title{
A simple method for the quantitative analysis of urinary delta-aminol evulinic acid to evaluate lead absorption
}

\author{
OSAMU WADA, KOHEI TOYOKAWA, \\ GUMPEI URATA, YUZO YANO, AND KIKU NAKAO \\ The Department of Hygiene and Preventive Medicine, and the Third Department of \\ Internal Medicine, Faculty of Medicine, University of Tokyo, Bunkyo-ku, Tokyo, Japan
}

\begin{abstract}
Wada, O., Toyokawa, K., Urata, G., Yano, Y., and Nakao, K. (1969). Brit. J. industr. Med., 26, 240-243. A simple method for the quantitative analysis of urinary delta-aminolevulinic acid to evaluate lead absorption. A procedure is given for the rapid, quantitative determination of urinary delta-aminolevulinic acid (ALA). Interfering substances are removed by $n$-butanol extraction. After pyrrole formation with ethyl acetoacetate, Ehrlich's reagent is added to produce the chromophore, which is then extracted with chloroform and measured spectrophotometrically or by comparison of the depth of colour with standard colour solutions. The recoveries were about $91 \%$ and the results agreed well with those obtained using ionexchange column chromatography $(r=0.985)$. This assay is simple, dependable, and suitable for large-scale screening of industrial workers exposed to lead poisoning, because the critical level of urinary ALA ( $20 \mathrm{mg}$./1. urine), which indicates dangerous lead absorption, gives a convenient absorbance.
\end{abstract}

Since Haeger-Aronsen (1960) established that there is an increase of urinary delta-aminolevulinic acid (ALA), an intermediate product in porphyrin biosynthesis, after abnormal lead exposure, the determination of urinary ALA has been considered one of the most reliable ways of assessing exposure to lead (Cramér and Selander, 1965; Gibson, Mackenzie, and Goldberg, 1968; Nakao, Wada, and Yano, 1968). However, the previously described method for the determination of urinary ALA (Mauzerall and Granick, 1956; Urata and Granick, 1963) depends on purification by ion-exchange chromatography, which is very time-consuming when applied to large numbers of specimens, though recently Davis and Andelman (1967) have developed a simple modification.

The method described below is based upon two purification steps using extraction by organic solvents without resorting to column chromatography. Simple extraction has proved adequate for the removal of most of the interfering substances, making the method suitable as a rapid, inexpensive and accurate screening procedure for the routine, quantitative determination of urinary ALA.

\section{Materials and methods}

All reagents were of reagent-grade purity unless otherwise specified. Standard ALA was obtained from the Sigma Chemical Company, London, S.W.6.

\section{Reagents}

$n$-Butanol; chloroform; aqueous acetic acid $(20 \%$ by vol.); sodium phosphate buffer (1 $\mathrm{M}, p \mathrm{H} 6.8)$; ethyl acetoacetate in sodium phosphate buffer $(1 / 20, v / v)$, Ehrlich's reagent, i.e., p-dimethylaminobenzaldehyde (4 g.), acetic acid (128 ml.), perchloric acid (70\%, $40 \mathrm{ml}$.), mercuric chloride $(0.2 \mathrm{M}, 10 \mathrm{ml}$.) $\mathrm{HCl}$ (conc., $40 \mathrm{ml}$.).

\section{Principle of the method}

Urobilinogen, other Ehrlich-positive substances, and some substances which interfere with the coupling reaction 
were separated from ALA by extracting weakly acidified urine with $n$-butanol. ALA was converted to its pyrrole by reaction with ethyl acetoacetate at $100^{\circ}$ at $p \mathrm{H} 6.8$. The pyrrole reacted with Ehrlich's reagent to form a red colour, which was extracted with chloroform, leaving other Ehrlich-positive substances in the water phase.

\section{Procedure}

To $2 \mathrm{ml}$. of urine were added $2 \mathrm{ml}$. of $20 \%$ acetic acid solution and $8 \mathrm{ml}$. of $n$-butanol. The solution was thoroughly mixed by vigorous shaking. After standing awhile, $0.5 \mathrm{ml}$. of aqueous phase was pipetted into each of two test tubes, to one of which $1.5 \mathrm{ml}$. of sodium phosphate buffer containing ethyl acetoacetate were added. To the other tube, which was run as a blank, were added $1.5 \mathrm{ml}$. of sodium phosphate buffer alone. The tubes were heated in a boiling water bath for 10 minutes. After cooling, $2 \mathrm{ml}$. of Ehrlich's reagent were added. Ten minutes later, $4 \mathrm{ml}$. of chloroform were added, and the mixture was shaken well. Elevated ALA concentrations were indicated by a reddish colour in chloroform, while normal concentrations usually gave only faint yellow or faint red colours. After 5 to $30 \mathrm{~min}$. the absorbance of the chloroform phase was read at $556 \mathrm{~nm}$. against the blank.

\section{Recovery tests}

These were carried out on urine specimens to which known quantities of ALA had been added. The ALA already in the urine before the addition of further ALA was estimated as described by Urata and Granick (1963). The method gave recoveries of about $90 \%$.

\section{Results and discussion}

\section{Recovery tests}

Urine usually contains many substances which react with Ehrlich's reagent to form a red colour, and also contains some substances which interfere with the pyrrole formation or the aldehyde reaction.

Recovery of known amounts of ALA from 10 to $35 \mathrm{mg}$./litre of urine, were about $90 \%$, as against 52 to $78 \%$ when the extraction with $n$-butanol was omitted (Table).

\section{Specificity}

Figure 1 shows the absorption curves for the final chloroform extract obtained from the urine of workers exposed to lead in comparison with that from authentic ALA. Small amounts of Ehrlichpositive substances which escaped the $n$-butanol extraction formed a red colour on addition of Ehrlich's reagent, but this never entered the chloroform phase. The only substance which behaved like ALA was aminoacetone. Urinary aminoacetone levels in lead poisoning have been reported to be entirely normal (Druyan and Haeger-Aronsen, 1964) and only range from 0 to $1 \mathrm{mg}$./litre of urine, whereas the critical level of urinary ALA which indicates dangerous lead absorption is considered to be $20 \mathrm{mg}$./litre of urine (Gibson et al., 1968).

The specificity is good enough to justify the use of the method for screening for lead poisoning.

\section{Sensitivity and reproducibility}

ALA is not extracted by $n$-butanol and it could be concentrated in the aqueous phase by repeated extraction with $n$-butanol, which dissolved appreciable quantities of water. Consequently, successive extractions with $n$-butanol also increased the sensitivity. The relative volumes of urine and

TABLE

Effect of $n$-Butanol Extraction on Absorbance at $556 \mathrm{~nm}$. AND the Recovery of AlA

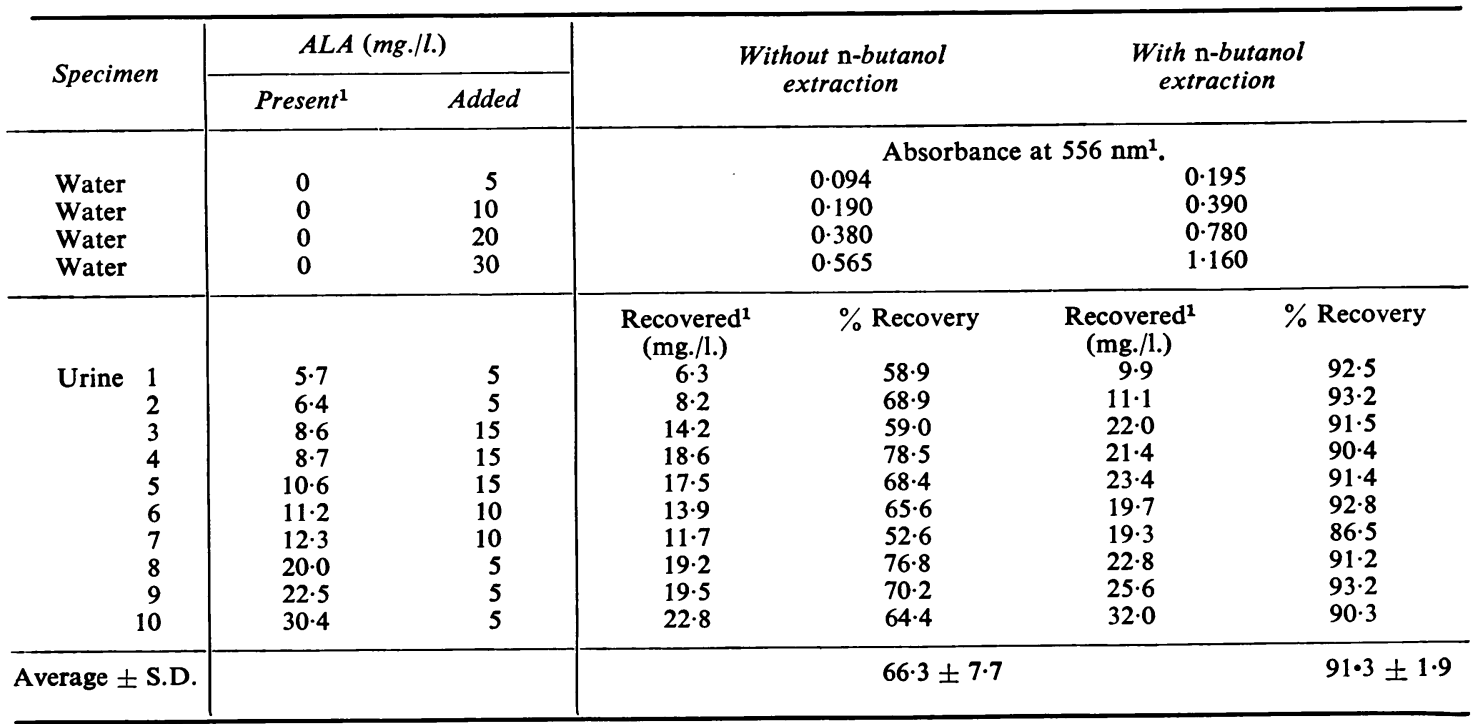

${ }^{1}$ Averages of duplicate analyses. 


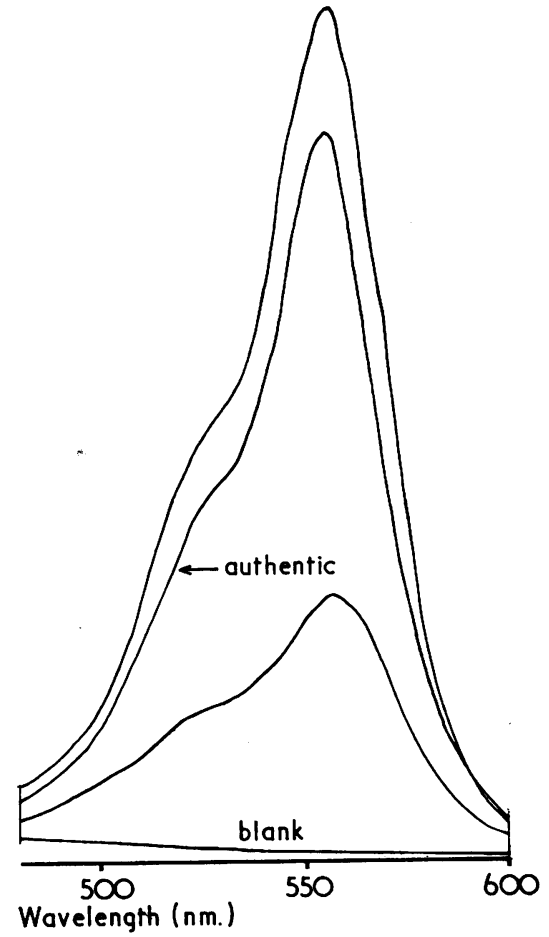

FIG. 1. Absorption curves for authentic and urinary ALA in chloroform. The blank was pure chloroform.

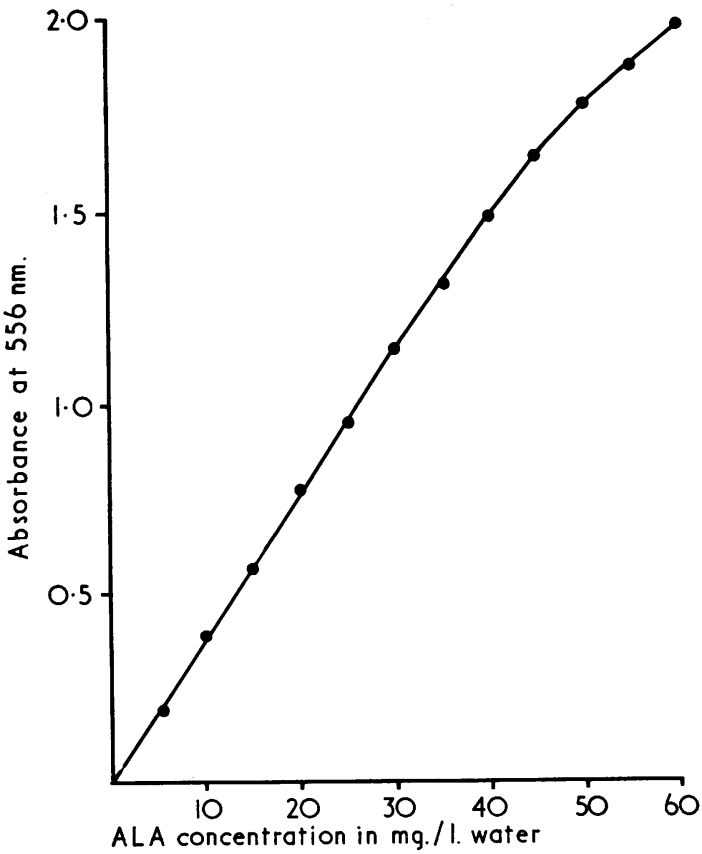

FIG. 2. Relationship between ALA concentration and absorbance at $556 \mathrm{~nm}$. ALA standards were prepared by dissolving ALA-hydrochloride in $0.1 \mathrm{M}$ acetate buffer, $p \mathrm{H} 4 \cdot 6$. $n$-butanol recommended here were chosen to give an absorbance of about 0.8 with urine containing the critical concentration of ALA, i.e., $20 \mathrm{mg}$./litre, as this is convenient for naked-eye comparisons.

The absorbance at $556 \mathrm{~nm}$. varied linearly with concentration up to $30 \mathrm{mg}$./litre when standard ALA solutions were analysed (Fig. 2). The standard error of a single estimation calculated from duplicate estimations on 20 urine specimens containing 5 to $48 \mathrm{mg}$./litre was $0.6 \mathrm{mg}$./litre.

Comparison with the method using ion-exchange column chromatography

Figure 3 shows a comparison between the values obtained with the method described above and the standard method of Urata and Granick (1963) on 25 samples of urine obtained from workers exposed to lead. A good agreement was obtained between the values obtained with two methods, especially above $5 \mathrm{mg}$. ALA/litre urine.

\section{Screening test}

As the optical density at $556 \mathrm{~nm}$. of the blanks (Fig. 1) never exceeded 0.025 and their colour was faint yellow, the depth of colour in the sample tubes could be conveniently estimated by comparison of the depth of colour with similar tubes containing standard coloured solutions. A series of stable standard coloured solutions was prepared by dissolving phenol red powder in weakly alkaline solution, $1 \mathrm{~m}$ potassium phosphate buffer, $p \mathrm{H} \mathrm{8.4}$. These were made up to match the colours given by known amounts of ALA in the full procedure and could then be used to find concentrations of ALA

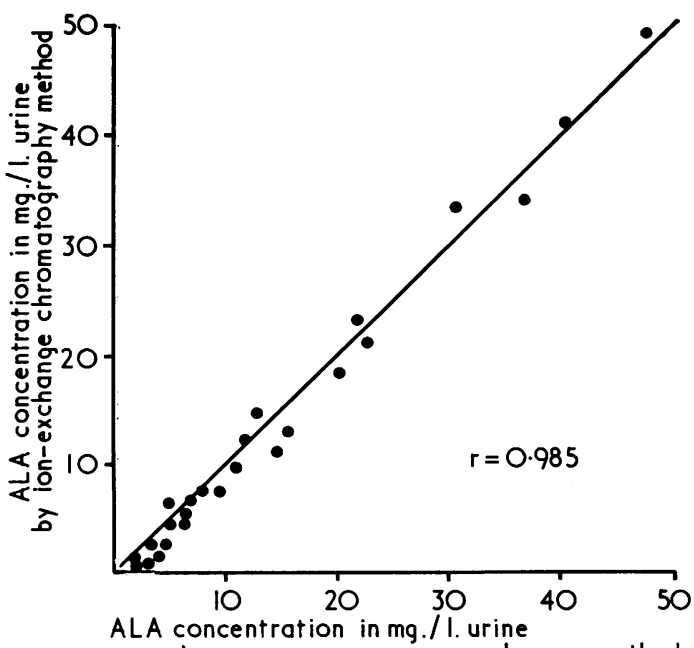

by new method

FIG. 3. Comparison of the Urata and Granick (1963) and the present method for determining ALA in urine. Urine specimens were obtained from workers exposed to lead. 
This study was supported in part by an N.I.H. Research Grant and in part by a Mishima Kaiun Foundation Research Grant.

\section{References}

Cramér, K., and Selander, S. (1965). Studies in lead poisoning. Comparison between different laboratory tests. Brit. J. industr. Med., 22, 311-314.

Davis, J. R., and Andelman, S. L. (1967). Urinary delta-aminolevulinic acid (ALA) levels in lead poisoning. Arch. environm. Hlth, 15, 53-59.

Druyan, R., and Haeger-Aronsen, B. (1964). Aminoacetone excretion in porphyrias and in chronic lead intoxication. Scand. J. clin. Lab. Invest., 16, 498-502.
Gibson, S. L. M., Mackenzie, J. C., and Goldberg, A. (1968). The diagnosis of industrial lead poisoning. Brit. J. industr. Med., 25 , 40-51.

Haeger-Aronsen, B. (1960). Studies on urinary excretion of deltaaminolevulinic acid and other haem precursors in lead workers and lead-intoxicated rabbits. Scand. J. clin. Lab. Invest., 12, Suppl. 47.

Mauzerall, D., and Granick, S. (1956). The occurrence and determination of delta-aminolevulinic acid and porphobilinogen in urine. J. biol. Chem., 219, 435-446.

Nakao, K., Wada, O., and Yano, Y. (1968). Delta-aminolevulinic acid dehydratase activity in erythrocytes for the evaluation of lead poisoning. Clin. chim. Acta, 19, 319-325.

Urata, G., and Granick, S. (1963). Biosynthesis of a-aminoketones and metabolism of aminoacetone. J. biol. Chem., 238, 811-820.

Received for publication September 30, 1968. 